\title{
AFFINE AND PROJECTIVE LINES OVER ONE-DIMENSIONAL SEMILOCAL DOMAINS
}

\author{
CHANDNI SHAH \\ (Communicated by Wolmer V. Vasconcelos)
}

\begin{abstract}
We characterize those partially ordered sets that can occur as the spectra of polynomial rings over one-dimensional semilocal (Noetherian) domains. We also determine the posets that can occur as projective lines over one-dimensional semilocal domains.
\end{abstract}

\section{INTRODUCTION AND PRELIMINARIES}

In $[W], R$. Wiegand shows that the structure of $\operatorname{Spec}(\mathbb{Z}[x])$ is much simpler than the structure of $\operatorname{Spec}(\mathbb{Q}[y, x])$ even though $\operatorname{Spec}(\mathbb{Z}) \cong \operatorname{Spec}(\mathbb{Q}[y])$. Here we view $\operatorname{Spec}(R)$ as the partially ordered set of prime ideals of $R$. However, Heinzer and S.Wiegand in $[\mathrm{HW}]$ show that for a countable one-dimensional semilocal domain $R, \operatorname{Spec}(R[x])$ is one of two types depending on whether or not $R$ is Henselian. In [HLW], Heinzer, Lantz and S.Wiegand extend the work in [HW] to analyze the structure of the projective line $\operatorname{Proj}(R[s, t])$. In $\S 2$, we generalize the characterization of $\operatorname{Spec}(R[x])$ in $[\mathrm{HW}]$ to include one-dimensional semilocal domains of arbitrary cardinality. In $\S 3$, we use the result of $\S 2$ to generalize the characterization of $\operatorname{Proj}(R[s, t])$ in [HLW] to one-dimensional semilocal domains of arbitrary cardinality. We note that the proofs in this paper are modifications of proofs in $[\mathrm{HW}]$ and $[\mathrm{HLW}]$. For results on the spectra of higher dimensional polynomial rings see $[\mathrm{M} 2, \mathrm{~S}, \mathrm{MS}]$.

Throughout this paper, $R$ denotes a Noetherian domain. In order to simplify our discussion we use the following terminology:

Definition 1.1. Let $P$ be a prime ideal in an integral domain $R$ and let $\mathbf{p}$ be a prime ideal of $R[x]$ such that $\mathbf{p} \cap R=P$. If $\mathbf{p} \neq P R[x]$, then $\mathbf{p}$ is called an upper to $P$ in $R[x]$. If the upper $\mathbf{p}$ contains a monic polynomial, then $\mathbf{p}$ is called an integral upper to $P$. If $\mathbf{p}$ is of the form $\mathbf{p}=(P, x-a)$ for some $a \in R$, then $\mathbf{p}$ is called a linear integral upper.

For elementary facts about uppers, see $[\mathrm{K}, \S 1-5]$.

In $\S 3$ we discuss the projective line $X$ over $R$. For convenience we use two interpretations of the projective line:

Received by the editors August 30, 1994.

1991 Mathematics Subject Classification. Primary 13A17,13B25,13E05,13H99,13J15.

Key words and phrases. Prime spectrum, Henselian ring, polynomial ring, projective line, discrete valuation domain. 
1. $X=\operatorname{Proj}(R[s, t])$, the set of relevant homogeneous primes in the polynomial ring in two indeterminates over $R$, and

2. $X$ is the union of the affine lines $\operatorname{Spec}(R[x])$ and $\operatorname{Spec}(R[1 / x])$, where $x=s / t$. Note that the only elements in the second affine line that are not in the first are the height-one prime $(1 / x) R[1 / x]$ and the height-two maximals $\left(P_{i}, 1 / x\right) R[1 / x]$, and the two affine lines intersect in $\operatorname{Spec}(R[x, 1 / x])$.

We refer to the relevant homogeneous prime ideals of $R[s, t]$ as points of $X$. We use the following terminology when discussing points of $X$.

Definition 1.2. Let $P$ be a prime ideal in an integral domain $R$ and let $\mathbf{p}$ be a point of $X=\operatorname{Proj}(R[s, t])$ such that $\mathbf{p} \cap R=P$. If $\mathbf{p} \neq P R[s, t]$, then $\mathbf{p}$ is called a projective upper to $P$ in $R[s, t]$. If the upper $\mathbf{p}$ contains a homogeneous polynomial $f$ such that the highest power of one of $s, t$ has coefficient 1 , then $\mathbf{p}$ is called a projective integral upper to $P$. If $\mathbf{p}$ is of the form $\mathbf{p}=(P, a s-b t)$ for $a, b \in R$ with one of $a, b=1$, then $\mathbf{p}$ is called a linear projective integral upper.

For elementary facts about graded rings and the homogeneous spectrum of $R[s, t]$ see $[\mathrm{Ku}, \S 1.5]$.

\section{The AfFine Line $\operatorname{Spec}(R[x])$}

We utilize the following notation used by Heinzer, Lantz and Wiegand [HLW] for discussing partially ordered sets:

For $u$ an element and $T$ a subset of a partially ordered set $U$ of finite dimension, let

$$
\begin{gathered}
G(u)=\{w \in U \mid w>u\}, \quad L(u)=\{w \in U \mid w<u\}, \\
L_{e}(T)=\{x \in U \mid G(x)=T\} .
\end{gathered}
$$

Let $\mathcal{M}(U)$ denote the set of elements of $U$ of maximal height.

Definition 2.1. Given infinite cardinalities $k_{1}, \ldots, k_{n} \leq r$, a partially ordered set $U$ is called affine of type $\left(r, k_{1}, \ldots, k_{n}\right)$ or $\mathbb{A}\left(r, k_{1}, \ldots, k_{n}\right)$ provided:

(P0) $|U|=r$.

(P1) $U$ has a unique minimal element $u_{0}$.

(P2) $U$ has dimension 2.

(P3) There exist $n$ height-one elements $u_{1}, \ldots, u_{n}$ (called special elements) satisfying

(i) $G\left(u_{1}\right) \cup G\left(u_{2}\right) \cup \cdots \cup G\left(u_{n}\right)=\mathcal{M}(U)$.

(ii) $G\left(u_{i}\right) \cap G\left(u_{j}\right)=\emptyset$ for $i \neq j$.

(iii) $\left|G\left(u_{i}\right)\right|=k_{i}$ for $i=1, \ldots, n$.

(P4) For each non-special height-one element $u, G(u)$ is finite (possibly $\emptyset$ ).

(P5) For each finite subset $T$ (including $\emptyset$ ) of $\mathcal{M}(U),\left|L_{e}(T)\right|=r$.

Remark. If $r, k_{1}, \ldots, k_{n}$ are countably infinite, then $\mathbb{A}\left(r, k_{1}, \ldots, k_{n}\right)$ is just $C \mathbb{Z}(n) P$ in the terminology used by $[\mathrm{HW}]$.

Definition 2.2. Given infinite cardinalities $k_{1} \leq r$, a partially ordered set $U$ is called Henselian affine of type $\left(r, k_{1}\right)$ or $H \mathbb{A}\left(r, k_{1}\right)$ provided:

$(\mathrm{P} 0)-(\mathrm{P} 4)$ of $\mathbb{A}\left(r, k_{1}\right)$ hold.

(P5) If $T$ is a finite subset of $\mathcal{M}(U)$, then $L_{e}(T)=\emptyset$ if $|T|>1$, and $\left|L_{e}(T)\right|=r$ if $|T| \leq 1$. 
Remark. If $r, k_{1}$ are countably infinite, then $H \mathbb{A}\left(r, k_{1}\right)$ is just $C H P$ in the terminology used by [HW].

Proposition 2.3. (1) If $U$ and $V$ are both $\mathbb{A}\left(r, k_{1}, \ldots, k_{n}\right)$, then $U$ is order isomorphic to $V$.

(2) If $U$ and $V$ are both $H \mathbb{A}\left(r, k_{1}\right)$, then $U \cong V$.

Proof. We define an order-preserving bijection $f$ from $U$ to $V$. For $i=0,1, \ldots, n$, let $f$ map $u_{i}$ to $v_{i}$ respectively. (Here $u_{0}, v_{0}$ are the unique minimal elements of $U, V$ respectively and $u_{i}, v_{i}$ are the special elements of $U, V$ respectively such that $\left|G\left(u_{i}\right)\right|=\left|G\left(v_{i}\right)\right|=k_{i}$ for $i=1, \ldots, n$.) For each $i$, let $f$ take the elements of $G\left(u_{i}\right)$ to elements of $G\left(v_{i}\right)$ (via any set bijection). It remains to define $f$ on non-special height-one elements of $U$. Note that the non-special height-one elements of $U$ can be partitioned into a collection of sets $\left\{L_{e}(T) \mid T\right.$ is a finite subset of $\left.\mathcal{M}(U)\right\}$ where in the Henselian case $|T| \leq 1$. Let $f$ send elements of $L_{e}(T)$ to $L_{e}(f(T)$ ) (again using any set bijection). It follows easily that $f$ gives an order isomorphism from $U$ to $V$.

Theorem 2.4. Let $U$ be a partially ordered set. Let $c$ denote the cardinality of $\mathbb{R}$ and let $r, k_{1}, \ldots, k_{n}$ be infinite cardinal numbers. Then

(i) $U \cong \operatorname{Spec}(R[x])$ for some one-dimensional semilocal non-Henselian domain $\left(R, P_{1}, \ldots, P_{n}\right)$ with $|R|=r$ and $\left|\frac{R}{P_{i}}[x]\right|=k_{i}$ for each $i$ if and only if $U$ is $\mathbb{A}\left(r, k_{1}, \ldots, k_{n}\right)$ and either (a) $r \leq c$ or (b) $k_{1}=\cdots=k_{n}=r>c$.

(ii) $U \cong \operatorname{Spec}(R[x])$ for some one-dimensional local Henselian domain $\left(R, P_{1}\right)$ where $r=|R|$ and $k_{1}=\left|\frac{R}{P_{1}}[x]\right|$ if and only if $U$ is $H \mathbb{A}\left(r, k_{1}\right)$ and either (a) $r \leq c$ or $(\mathrm{b}) k_{1}=r>c$.

Remark. If $R / P_{i}$ is infinite, then $\left|\frac{R}{P_{i}}[x]\right|=\left|\frac{R}{P_{i}}\right|$.

Before we can prove Theorem 2.4, we need the following lemmas and propositions.

Lemma 2.5. If $(R, M)$ is a local domain, then $|R| \leq \sup (|R / M|, c)$. Thus if $|R|>c$, then $|R / M|=|R|$.

Proof. For $i \geq 0$, let $R_{i}$ be a complete set of representatives in $R$ of each residue class of $M^{i} / M^{i+1}$. We define a set map $\Psi: R \rightarrow \prod_{i=0}^{\infty} R_{i}$. For $a \in R$, let $\Psi(a)=$ $\left(a_{0}, a_{1}, \ldots\right)$, where $a_{i} \in R_{i}$ is such that $a_{i} \equiv a-\sum_{j=0}^{i-1} a_{j} \bmod M^{i+1}$. Note that $\Psi$ is one-one since $\bigcap_{i=0}^{\infty} M^{i}=0$. Since $M^{i} / M^{i+1}$ is a finite-dimensional vector space over $R / M,\left|M^{i} / M^{i+1}\right|=\left|R_{i}\right|$ is either finite or equal to $|R / M|$. Thus $|R| \leq$ $|R / M|^{\aleph_{0}}=\sup (|R / M|, c)$.

Lemma 2.6 [HM, Theorem 1.2]. For $k \geq 2$, let $P_{1}, \ldots, P_{k}$ be non-zero primes (not necessarily distinct) of a non-Henselian Noetherian domain $R$. Then there exists a finitely generated integral extension domain $R^{\prime}$ of $R$ containing distinct, pairwise comaximal primes $P_{1}^{\prime}, \ldots, P_{k}^{\prime}$ lying over $P_{1}, \ldots, P_{k}$ respectively.

Lemma 2.7 [S, Lemma 3.7]. Let $\mathbf{p}_{1}, \ldots, \mathbf{p}_{k}$ be integral uppers in $R[x]$ ( $R$ not necessarily Noetherian). There exists a finitely generated integral extension domain $R^{\prime}$ of $R$ such that if $\mathbf{p}^{\prime}$ is any prime in $R^{\prime}[x]$ with $\mathbf{p}^{\prime} \cap R[x]=\mathbf{p}_{i}$, then $\mathbf{p}^{\prime}$ is a linear integral upper. 
Proposition 2.8. Let $(R, M)$ be a local Henselian domain. Let $\mathbf{k}$ be an upper to (0). Then one of the following holds: (a) $\mathbf{k}$ is contained in $M R[x]$, (b) $\mathbf{k}$ is contained in no upper to $M$ or (c) $\mathbf{k}$ is an integral upper to (0) and is contained in a unique upper to $M$.

Proof. Suppose that (a) and (b) are false. We must show that (c) holds. Let $F$ be the quotient field of $R$. Now $\mathbf{k}$ is contained in at least one upper $\mathbf{m}$ to $M$. Also $\mathbf{k}=\alpha(x) F[x] \cap R[x]$ where $\alpha(x)$ is a monic irreducible polynomial in $F[x]$. Let $u$ be a root of $\alpha(x)$. Then $\mathbf{k}$ is the kernel of the canonical map $R[x] \rightarrow R[u]$. We show that $u$ is in fact integral over $R$. Let $S$ be the integral closure of $R$ in $R[u]$. Note that $u$ is in the quotient field of $S$ since $u$ is algebraic over $R$. Also $S$ has a unique maximal ideal $N$ since $S$ is an integral extension of $R$ [N, 30.5]. We note that $R[u]=S[u]$. Let $Q$ be the image of $\mathbf{m} / \mathbf{k}$ under the isomorphism $R[x] / \mathbf{k} \cong R[u]=S[u]$. Under the canonical embedding $R \subset R[x] / \mathbf{k}$, we see that $\frac{\mathbf{m}}{\mathbf{k}} \cap R=M$; thus $Q \cap R=M$ and so $Q \cap S=N$. Now by assumption, $\mathbf{k} \nsubseteq \mathbb{M} R[x]$, so $u$ must satisfy some polynomial in $R[x] \subseteq S[x]$ with at least one coefficient which is not in $M$ and hence not in $N$. By the $u, u^{-1}-$ Lemma $[\mathrm{K}, \S 1-6$, Exercise 31] either $u$ or $u^{-1}$ is in $S$. If $u \notin S$, then $u^{-1} \in N$ which implies $u^{-1} \in Q$, which contradicts that $Q$ is a proper ideal of $S[u]$. Therefore $u \in S$ so that $S=S[u]=R[u]$. Thus we see that $u$ is integral over $R$ and so $\mathbf{k}$ contains a monic polynomial and thus is an integral upper to (0). Now if $\mathbf{k} \subseteq \mathbf{m}^{\prime}$ where $\mathbf{m}^{\prime}$ is an upper to $M$ distinct from $\mathbf{m}$, then in the integral extension $R \subseteq R[x] / \mathbf{k}$ we would have two distinct maximal ideals $\mathbf{m} / \mathbf{k}$ and $\mathbf{m}^{\prime} / \mathbf{k}$ which contradicts the fact that $R[x] / \mathbf{k}$ must be local $[\mathrm{N}$, 43.16].

Proposition 2.9. Let $R$ be a non-Henselian one-dimensional semilocal domain. Let $\mathbf{p}_{1}, \ldots, \mathbf{p}_{k}$ be height-two primes of $R[x]$. Then there exist $|R|$ height-one primes $\mathbf{k}$ such that $\mathbf{k} \subseteq \mathbf{p}_{i}$ for each $i$, but $\mathbf{k} \nsubseteq \mathbf{m}$ for every height-two maximal ideal $\mathbf{m}$ of $R[x]$ with $\mathbf{m} \notin\left\{\mathbf{p}_{1}, \ldots, \mathbf{p}_{k}\right\}$.

Proof. For each $i=1, \ldots, k$, let $P_{i}=\mathbf{p}_{i} \cap R$. Then $P_{1}, \ldots, P_{k}$ are non-zero primes of $R$ and each $\mathbf{p}_{i}$ is an integral upper to $P_{i}$ respectively. By Lemma 2.6, there exists a finitely generated integral extension domain $R^{\prime}$ of $R$ with distinct (necessarily maximal) primes $P_{1}^{\prime}, \ldots, P_{k}^{\prime}$ lying over $P_{1}, \ldots, P_{k}$, respectively. By going up we can find integral uppers $\mathbf{p}_{i}^{\prime}$ in $R^{\prime}[x]$ lying over $\mathbf{p}_{i}$ respectively. By going to yet another finitely generated integral extension domain $S$ of $R^{\prime}$ we can find primes $\mathbf{q}_{i}$ in $S[x]$ lying over $\mathbf{p}_{i}^{\prime}$ such that $\mathbf{q}_{i}=\left(Q_{i}, x-a_{i}\right)$ for some $a_{i} \in S$, where $Q_{i}=\mathbf{q}_{i} \cap S$ (Lemma 2.7). Since $S$ is a finitely generated integral extension domain of $R$, we note that $S$ is also a non-Henselian semilocal domain. For convenience, we label the non-zero primes of $S$ as $Q_{1}, \ldots, Q_{k}, Q_{k+1}, \ldots, Q_{m}$. Let $E$ be the quotient field of $S$. We find $|S|$ uppers $\mathbf{l}_{\mu}$ to (0) with $\mathbf{l}_{\mu} \subseteq \mathbf{q}_{i}$ for each $i$ but $\mathbf{l}_{\mu} \nsubseteq \subseteq \mathbf{n}$ for every height-2 maximal $\mathbf{n} \notin\left\{\mathbf{q}_{\mathbf{1}}, \ldots, \mathbf{q}_{\mathbf{k}}\right\}$.

Using the Chinese Remainder Theorem we can find $a, b \in S$ such that

for $1 \leq i \leq k, a \equiv a_{i}$ and $b \equiv 1 \bmod Q_{i}$.

for $k+1 \leq i \leq m, a \equiv 1$ and $b \equiv 0 \bmod Q_{i}$.

For each element $\mu$ in the Jacobson radical of $S$, let $c_{\mu}=a+\mu$.

Remark. If $I$ is a non-zero ideal of $S$, then since $I=I S,|I|=|S|$. Therefore the Jacobson radical of $S$ has cardinality $|S|$.

Note that $c_{\mu} \equiv a_{i} \bmod Q_{i}$ for $1 \leq i \leq k$ and $c_{\mu} \equiv 1 \bmod Q_{i}$ for $k+1 \leq i \leq m$. 
Now $\mathbf{l}_{\mu}=\left(x-\frac{c_{\mu}}{b}\right) E[x] \cap S[x]$ is the unique minimal prime divisor of $\left(b x-c_{\mu}\right) S[x]$ since there does not exist any height-one prime in $S$ containing both $b, c_{\mu}$ [M1, Lemma 2]. We claim that $\left\{\mathbf{1}_{\mu}\right\}$ is the desired collection of uppers to (0). Suppose $\mathbf{l}_{\mu}$ is contained in some upper $\mathbf{q}$ to $Q_{i}$ for some $i$.

If $1 \leq i \leq k$, then $b x-c_{\mu} \equiv x-a_{i} \bmod Q_{i}$; thus $\left(Q_{i}, x-a_{i}\right) \subseteq \mathbf{q} \Rightarrow \mathbf{q}=\mathbf{q}_{i}$.

If $k+1 \leq i \leq m$, then $b x-c_{\mu} \equiv-1 \bmod Q_{i} \Rightarrow-1 \in \mathbf{q}$ which is a contradiction. We return to $R[x]$. Now the set $\left\{\mathbf{k}_{\mu} \mid \mathbf{k}_{\mu}=\mathbf{l}_{\mu} \cap R[x]\right\}$ has cardinality $|R|=|S|$, since $S$ is a finitely generated integral extension of $R$. Clearly $\mathbf{k}_{\mu} \subseteq \mathbf{p}_{i}$ for each $i$. Now suppose $\mathbf{k}_{\mu} \subseteq \mathbf{m}$ for some upper $\mathbf{m}$. Since $\mathbf{l}_{\mu}$ in $S[x]$ lies over $\mathbf{k}_{\mu}$, by going up there exists a maximal ideal $\mathbf{n}$ of $S[x]$ lying over $\mathbf{m}$ such that $\mathbf{l}_{\mu} \subseteq \mathbf{n}$. But by construction of $\mathbf{l}_{\mu}, \mathbf{n}=\mathbf{q}_{i}$ for some $i$. Therefore $\mathbf{m}=\mathbf{q}_{i} \cap R[x]=\mathbf{p}_{i}$. Hence $\left\{\mathbf{k}_{\mu}\right\}$ is our desired collection.

Proof of Theorem 2.4. We first show that if $\left(R, P_{1}, \ldots, P_{n}\right)$ is a one-dimensional semilocal domain with $|R|=r$ and $\left|\frac{R}{P_{i}}[x]\right|=k_{i}$, then $\operatorname{Spec}(R[x])$ satisfies $(\mathrm{P} 0)-(\mathrm{P} 4)$ of $\mathbb{A}\left(r, k_{1}, \ldots, k_{n}\right)$.

Since $|R[x]|=|R|=r$, if $T$ is any subset of $\operatorname{Spec}(R[x])$, then $|T| \leq r$. Since $(x-a) R[x]$ is prime in $R[x]$ for every $a \in R, \operatorname{Spec}(R[x])$ satisfies (P0). Of course (0) is the unique minimal prime of $R[x]$. (P2) follows from the fact that the Krull dimension of $R[x]$ is 2 . For (P3), let each $u_{i}$ be $P_{i} R[x]$. If $\mathbf{m}$ is a maximal ideal of height 2 , then $\mathbf{m}$ is an upper to $P_{i}$ for some $i$ so that $\mathbf{m} \in G\left(u_{i}\right)$. Also $G\left(u_{i}\right)$ corresponds to the maximal ideals of $\frac{R}{P_{i}}[x]$, thus $\left|G\left(u_{i}\right)\right|=k_{i}$. For (P4), note that for $u$ non-special height-one, $G(u)$ is a finite union of all the $G(u) \cap G\left(u_{i}\right)$ which must be finite. (Since in the Noetherian ring $R[x]$, there are only finitely many primes minimal over $u+u_{i}$.)

For (P5) of (2.1) and (2.2), let $T$ be a finite subset of $\mathcal{M}(\operatorname{Spec}(R[x]))$. Suppose $T$ is empty. For each $\beta$ in the Jacobson radical of $\mathrm{R},(\beta x-1) K[x] \cap R[x]$ is a maximal height-one prime of $R[x]$; thus $\left|L_{e}(T)\right|=r$. If $T$ is a non-empty finite collection of height-2 maximal ideals of $R[x]$, Theorem 2.4 breaks up into two cases depending on whether or not $R$ is Henselian:

For (i) of Theorem 2.4, suppose $T=\left\{\mathbf{p}_{1}, \ldots, \mathbf{p}_{k}\right\}$. Then by Proposition 2.9, there are $r$ height-one primes $\mathbf{k}$ such that $\mathbf{k} \subseteq \mathbf{m}$ for $\mathbf{m}$ a height- 2 maximal ideal if and only if $\mathbf{m} \in\left\{\mathbf{p}_{1}, \ldots, \mathbf{p}_{k}\right\}$. Thus for each finite subset $T$ of $\mathcal{M}(\operatorname{Spec}(R[x]))$, $\left|L_{e}(T)\right|=r$. Thus (P5) of $\mathbb{A}\left(r, k_{1}, \ldots, k_{n}\right)$ is satisfied.

For (ii), if $|T|>1$, then by Proposition $2.8, L_{e}(T)=\emptyset$. Suppose $T=\{\mathbf{p}\}$. By Lemma 2.7 there exist a finitely generated extension domain $S$ (necessarily Henselian) of $R$ and a prime $\mathbf{q}$ of $S[x]$ lying over $\mathbf{p}$ such that $\mathbf{q}=\left(Q_{1}, x-a\right)$ for some $a \in S$, where $Q_{1}$ is the unique maximal ideal of $S$. For each $\alpha \in Q_{1}$, let $\mathbf{l}_{\alpha}=(x-a+\alpha) S[x]$. Now as in the proof of Proposition 2.8, $\left\{\mathbf{k}_{\alpha}=\mathbf{l}_{\alpha} \cap R[x]\right\}$ is a collection of $r$ integral uppers to (0) with $\mathbf{k}_{\alpha} \subseteq \mathbf{p}$. Hence axiom (P5) of $H \mathbb{A}\left(r, k_{1}\right)$ holds.

We now prove the cardinality restrictions. If $r>c$, we must show that each $k_{i}=$ $r$. Localize $R$ at $P_{i}$ to obtain a local domain $\left(R_{P_{i}}, P_{i} R_{P_{i}}\right)$. Since $\left|R_{P_{i}}\right|=|R|>c$, by Lemma 2.5, $r=|R|=\left|R_{P_{i}}\right|=\left|R_{P_{i}} / P_{i} R_{P_{i}}\right|=\left|R / P_{i}\right|=\left|\frac{R}{P_{i}}[x]\right|=k_{i}$.

For the converse of (i), we construct a one-dimensional normal semilocal domain $\left(R, P_{1}, \ldots, P_{n}\right)$ with $|R|=r$ and $\left|R / P_{i}\right|=k_{i}$. We first deal with the case $r \leq c$. I am grateful to $R$. Heitmann for suggesting the technique used in constructing the following example. 
Example 2.10. Let $A$ be a set of cardinality $r$ and let $B_{1}, \ldots, B_{n}$ be subsets of $A$ of cardinality $k_{1}, \ldots k_{n}$ respectively (if $k_{i}=r$, choose $B_{i}=A$ ). Let $F=$ $\mathbb{Q}\left(z_{1}, \ldots z_{n},\left\{t_{\alpha}\right\}_{\alpha \in A}\right)$, where $z_{j}, t_{\alpha}$ are indeterminates. Thus $F$ is a field of cardinality $r$. Now $K_{i}=\mathbb{Q}\left(z_{1}, \ldots, z_{i-1}, z_{i+1}, \ldots, z_{n},\left\{t_{\alpha}\right\}_{\alpha \in B_{i}}\right)$ is a proper subfield of $F$ of cardinality $k_{i}$. Let $K_{i}^{*}=K_{i}[[y]]\left[y^{-1}\right]$. Either $k_{i}=r=c$ (in which case $\left.F=K_{i}\left(z_{i}\right)\right)$ or the transcendence degree of $K^{*}$ over $K_{i}$ is $c$ (since $\left|K^{*}\right|=c>k_{i}$ ). In any case we can extend the inclusion map of $K_{i}$ into $K_{i}^{*}$ to an isomorphism $\phi_{i}: F \rightarrow K_{i}^{*}$ onto $F_{i}^{*}=\phi_{i}(F)$ such that $\phi_{i}\left(z_{i}\right)=y$. By [K, Theorem 99], it follows that $F_{i}^{*} \cap K_{i}[[y]]$ is a DVR of $F_{i}^{*}$ with maximal ideal $y K_{i}[[y]] \cap F_{i}^{*}$. By pulling back we get that $V_{i}=\phi_{i}^{-1}\left(K_{i}[[y]]\right)$ is a DVR of $F$ with maximal ideal $N_{i}=\phi_{i}^{-1}\left(y K_{i}[[y]]\right)$ such that $\left|V_{i} / N_{i}\right|=\left|K_{i}[[y]] / y K_{i}[[y]]\right|=k_{i}$. Since $z_{i}$ is a unit in $V_{j}$ if and only if $i \neq j, V_{i} \not \subset V_{j}$ for $i \neq j$. Let $R=V_{1} \cap \cdots \cap V_{n}$ and let $P_{i}=N_{i} \cap R$. Then by [Ma, Theorem 12.2], $\left(R, P_{1} \ldots, P_{n}\right)$ has the desired properties.

We now assume $k_{1}=\cdots=k_{n}=r>c$.

Example 2.11. Let $F$ be a field of cardinality $r$. In $T=F[z]$, let $P_{i}=\left(z-a_{i}\right) F[z]$ where $z$ is an indeterminate and $a_{1}, \ldots, a_{n}$ are distinct elements of $F$. Localizing $T$ at the set $S=T-\left(P_{1} \cup \cdots \cup P_{n}\right)$ gives a normal semilocal non-Henselian domain $\left(T_{S}, P_{1} T_{S}, \ldots, P_{n} T_{S}\right)$ with the required cardinalities.

The converse of (ii) follows from the following:

Remark 2.12. Let $(R, M)$ be a normal local domain and let $\left(R^{*}, M^{*}\right)$ be the Henselization of $(R, M)$. Then $\left(R^{*}, M^{*}\right)$ is a local Henselian domain with $\left|R^{*}\right|=|R|$ and $R^{*} / M^{*}=R / M[\mathrm{~N}, \S 43]$.

\section{The Projective Line $\operatorname{Proj}(R[s, t])$}

Let $\left(R, P_{1}, \ldots, P_{n}\right)$ be a one-dimensional semilocal domain. We introduce two axiom systems analogous to the ones introduced in $\S 2$.

Definition 3.1. Given infinite cardinalities $k_{1}, \ldots, k_{n} \leq r$, a partially ordered set $U$ is called projective of type $\left(r, k_{1}, \ldots, k_{n}\right)$ or $\mathbb{P}\left(r, k_{1}, \ldots, k_{n}\right)$ provided:

(P0) $|U|=r$.

(P1) $U$ has a unique minimal element $u_{0}$.

(P2) $U$ has dimension 2.

(P3) There exist $n$ height-one elements $u_{1}, \ldots, u_{n}$ (called special elements) satisfying

(i) $G\left(u_{1}\right) \cup G\left(u_{2}\right) \cup \cdots \cup G\left(u_{n}\right)=\mathcal{M}(U)$.

(ii) $G\left(u_{i}\right) \cap G\left(u_{j}\right)=\emptyset$ for $i \neq j$.

(iii) $\left|G\left(u_{i}\right)\right|=k_{i}$ for $i=1, \ldots, n$.

(P4) For each non-special height-one element $u, G(u)$ is finite and $G(u) \cap G\left(u_{i}\right) \neq$ $\emptyset$ for each $1 \leq i \leq n$.

(P5) For each non-empty finite subset $T$ of $\mathcal{M}(U)$ such that $\left\{u_{1}, \ldots, u_{n}\right\} \subseteq$ $\bigcup\{L(t) \mid t \in T\},\left|L_{e}(T)\right|=r$.

Remark. If $r, k_{1}, \ldots, k_{n}$ are countably infinite, then $\mathbb{P}\left(r, k_{1}, \ldots, k_{n}\right)$ is just $\mathbf{P} C \mathbb{Z}(n) P$ in the terminology used by [HLW].

Definition 3.2. Given infinite cardinalities $k_{1} \leq r$, a partially ordered set $U$ is called Henselian projective of type $\left(r, k_{1}\right)$ or $H \mathbb{P}\left(r, k_{1}\right)$ provided: 
(P0)-(P4) of $\mathbb{P}\left(r, k_{1}\right)$ hold.

(P5) If $T$ is a finite subset of $\mathcal{M}(U)$, then $L_{e}(T)=\emptyset$ if $|T| \neq 1$, and $\left|L_{e}(\{t\})\right|=r$.

Remark. If $r, k_{1}$ are countably infinite, then $H \mathbb{P}\left(r, k_{1}\right)$ is just $\mathbf{P} C H P$ in the terminology used by [HLW].

Proposition 3.3. (1) If $U$ and $V$ are both $\mathbb{P}\left(r, k_{1}, \ldots, k_{n}\right)$, then $U \cong V$.

(2) If $U$ and $V$ are both $H \mathbb{P}\left(r, k_{1}\right)$, then $U \cong V$.

Proof. Note that the non-special height-one elements can be partitioned into a collection $\left\{L_{e}(T)\right\}$ for appropriate finite subsets $T$ of $\mathcal{M}(U)$. The proof is analogous to the proof of Proposition 2.3.

Theorem 3.4. Let $U$ be a partially ordered set. Let $r, k_{1}, \ldots, k_{n}$ be infinite cardinal numbers. Then

(i) $U \cong \operatorname{Proj}(R[s, t])$ for some one-dimensional semilocal non-Henselian domain $\left(R, P_{1}, \ldots, P_{n}\right)$ with $|R|=r$ and $\left|\frac{R}{P_{i}}[x]\right|=k_{i}$ for each $i$ if and only if $U$ is $\mathbb{P}\left(r, k_{1}, \ldots, k_{n}\right)$ and either (a) $r \leq c$ or (b) $k_{1}=\cdots=k_{n}=r>c$.

(ii) $U \cong \operatorname{Proj}(R[s, t])$ for some one-dimensional local Henselian domain $\left(R, P_{1}\right)$ where $r=|R|$ and $k_{1}=\left|\frac{R}{P_{1}}[x]\right|$ if and only if $U$ is $H \mathbb{P}\left(r, k_{1}\right)$ and either

(a) $r \leq c$ or (b) $k_{1}=r>c$.

Before we can prove Theorem 3.4, we need the following lemmas and proposition.

Remark 3.5. Let $S$ be an integral extension of $R$ and let $\mathbf{q}$ be a homogeneous prime in $S[s, t]$. Then $\mathbf{q} \cap R[s, t]$ is a homogeneous prime in $R[s, t]$. We also note that lying over and going up theorems also hold for homogeneous primes.

Lemma 3.6. Let $\mathbf{p}_{1}, \ldots, \mathbf{p}_{k}$ be a finite set of height-two points of $X$. Then there is a finitely generated integral extension $R^{\prime}$ of $R$ such that if $\mathbf{p}^{\prime}$ is any point in $X^{\prime}=\operatorname{Proj}\left(R^{\prime}[s, t]\right)$ lying over $\mathbf{p}_{i}$, then $\mathbf{p}^{\prime}$ is a linear projective integral upper.

Proof. This follows immediately by applying Lemma 2.7 , by considering the heighttwo points as maximal ideals in either $R[s / t]$ or $R[t / s]$.

Lemma 3.7. Let $\mathbf{k}$ be a projective upper to 0 in $X$. Then for each $i=1, \ldots, n$, there exists a projective upper $\mathbf{p}_{i}$ to $P_{i}$ containing $\mathbf{k}$.

Proof. See [HLW], proof of second part of (P5) of Lemma 2.4.

Proposition 3.8. Let $\left(R, P_{1}, \ldots, P_{n}\right)$ be a non-Henselian one-dimensional semilocal domain. Let $\mathbf{p}_{11}, \ldots, \mathbf{p}_{1 r_{1}}, \ldots, \mathbf{p}_{n 1}, \ldots, \mathbf{p}_{n r_{n}}$ be height-two points of $X$ such that each $r_{i} \geq 1$ and $\mathbf{p}_{i j} \cap R=P_{i}$. Then there exist $|R|$ height-one points $\mathbf{k}$ such that $\mathbf{k} \subseteq \mathbf{p}_{i j}$ for each $i=1, \ldots, n, j=1, \ldots, r_{i}$, but $\mathbf{k} \nsubseteq \mathbb{\mathbf { m }}$ for any height-two point $\mathbf{m}$ of $X$ with $\mathbf{m} \notin\left\{\mathbf{p}_{11}, \ldots, \mathbf{p}_{1 r_{1}}, \ldots, \mathbf{p}_{n 1}, \ldots, \mathbf{p}_{n r_{n}}\right\}$.

Proof. Note that the height-two points of $X$ are necessarily integral projective uppers. In light of Lemma 3.6, we replace $R$ by an integral extension of $R$ and get a (possibly larger) collection of all points in the projective line of the integral extension lying over some $\mathbf{p}_{i j}$. Thus we assume that each $\mathbf{p}_{i j}$ is a linear projective integral upper. By Lemma 2.6, there exists a finitely generated integral extension domain $S$ of $R$ with distinct (maximal) primes $P_{11}^{\prime}, \ldots, P_{n r_{n}}^{\prime}$ such that $P_{i j}^{\prime}$ lies over $P_{i}$. Relabel the non-zero primes of $S$ as $Q_{1} \ldots, Q_{m}$ and for $j=1, \ldots, m$, pick a linear projective integral upper $\mathbf{q}_{j}$ in such a way that $\left\{\mathbf{q}_{1}, \ldots, \mathbf{q}_{m}\right\} \cap R[s, t]=$ $\left\{\mathbf{p}_{11}, \ldots, \mathbf{p}_{n r_{n}}\right\}$. Thus we assume $Q_{1}, \ldots, Q_{m}$ are distinct non-zero primes of $S$ 
and for $j=1, \ldots, m, \mathbf{q}_{j}=\left(Q_{j}, a_{j} s-b_{j} t\right)$ where one of $a_{j}, b_{j}$ is 1 . We find $|S|$ projective uppers $\mathbf{l}_{\mu}$ to (0) with $\mathbf{l}_{\mu} \subset \mathbf{q}_{j}$ for each $j$ but $\mathbf{l}_{\mu} \not \subset \mathbf{n}$ for any height-2 maximal $\mathbf{n} \notin\left\{\mathbf{q}_{\mathbf{1}}, \ldots, \mathbf{q}_{\mathbf{m}}\right\}$.

By the Chinese Remainder Theorem we find elements $a, b$ in $S$ such that for each $j, a \equiv a_{j} \bmod Q_{j}$ and $b \equiv b_{j} \bmod Q_{j}$. For each element $\mu$ in the Jacobson radical of $S$, let $c_{\mu}=b-\mu$. Note that $c_{\mu} \equiv b \equiv b_{j} \bmod Q_{j}$. Now $\mathbf{l}_{\mu}=\left(a s-c_{\mu} t\right) S[s, t]$ is a linear projective upper to $(0)$, since $\bmod$ each $Q_{j}$, either $a$ or $c_{\mu}$ is congruent to 1 . We claim that $\left\{\mathbf{l}_{\mu}\right\}$ is the desired collection of projective uppers to (0). Suppose $\mathbf{l}_{\mu}$ is contained in a projective upper $\mathbf{q}$ to some $Q_{j}$. Then since $a s-c_{\mu} t \equiv a_{j} s-b_{j} t$ $\bmod Q_{j}, \mathbf{q}$ must contain $\left(Q_{j}, a_{j} s-b_{j} t\right)=\mathbf{q}_{j}$ which implies $\mathbf{q}=\mathbf{q}_{j}$.

We return to $R[s, t]$. Now the set $\left\{\mathbf{k}_{\mu} \mid \mathbf{k}_{\mu}=\mathbf{l}_{\mu} \cap R[s, t]\right\}$ has cardinality $|R|=|S|$, since $S$ is a finitely generated integral extension of $R$. It is not difficult to see that $\mathbf{k}_{\mu}$ is a projective upper to (0), i.e. a point in $X$. Clearly $\mathbf{k}_{\mu} \subseteq \mathbf{p}_{i j}$ for each $i, j$. Now suppose $\mathbf{k}_{\mu} \subset \mathbf{m}$ for some height-two point $\mathbf{m}$. Since $\mathbf{l}_{\mu}$ in $Y=\operatorname{Proj} S[s, t]$ lies over $\mathbf{k}_{\mu}$, by going up there exists a height-two point $\mathbf{n}$ of $Y$ lying over $\mathbf{m}$ such that $\mathbf{l}_{\mu} \subseteq \mathbf{n}$. But by construction of $\mathbf{l}_{\mu}, \mathbf{n}=\mathbf{q}_{j}$ for some $j=1, \ldots, m$. Therefore $\mathbf{m}=\mathbf{q}_{j} \cap R[s, t] \in\left\{\mathbf{p}_{11}, \ldots, \mathbf{p}_{n r_{n}}\right\}$. Hence $\left\{\mathbf{k}_{\mu}\right\}$ is our desired collection.

Proof of Theorem 3.4. In view of the proof of Theorem 2.4, it suffices to show that if $\left(R, P_{1}, \ldots, P_{n}\right)$ is a one-dimensional semilocal domain with $|R|=r$ and $\left|\frac{R}{P_{i}}[x]\right|=k_{i}$, then $\operatorname{Proj}(R[s, t])$ is (i) $\mathbb{P}\left(r, k_{1}, \ldots, k_{n}\right)$ if $R$ is not Henselian and (ii) $H \mathbb{P}\left(r, k_{1}\right)$ if $n=1$ and $R$ is Henselian. We first show $\operatorname{Proj}(R[s, t])$ satisfies $(\mathrm{P} 0)-$ $(\mathrm{P} 4)$ of $\mathbb{P}\left(r, k_{1}, \ldots, k_{n}\right)$. From the second definition of $X=\operatorname{Proj}(R[s, t])$ and the fact that there are only finitely many points of $X$ not in $R[x]=R[s / t]$, we see that most of the cardinality arguments of Theorem 2.4 carry over to $\operatorname{Proj}(R[s, t])$. Thus (P0) follows immediately. Of course (0) is the unique minimal element of $X$. Since $\operatorname{Spec}(R[s, t])$ has dimension 3 and the only height-three homogeneous primes in $R[s, t]$ are of the form $\left(P_{i}, s, t\right) R[s, t]$, which are irrelevant, it follows that $X$ has dimension 2. For (P3), we let $u_{i}$ be the points $P_{i} R[s, t]$, the rest follow easily from Theorem 2.4. (P4) follows from Lemma 3.7.

Let $T$ be a finite collection of height-two points of $X$ such that each $P_{i}$ is contained in some point in $T$. Again (P5) breaks up into two cases. For (i), suppose $T=\left\{\mathbf{p}_{11}, \ldots, \mathbf{p}_{n r_{n}}\right\}$. Then by Proposition 3.8, there are $r$ height-one points $\mathbf{k}$ such that $\mathbf{k} \subset \mathbf{m}$ for $\mathbf{m}$ a height-two point of $X$ if and only if $\mathbf{m} \in\left\{\mathbf{p}_{11}, \ldots, \mathbf{p}_{n r_{n}}\right\}$. Thus for each finite subset $T$ of $\mathcal{M}(X)$ with $\left\{u_{1}, \ldots, u_{n}\right\} \subseteq \bigcup\{L(t) \mid t \in T\},\left|L_{e}(T)\right|=r$.

For (ii), we first show that if $|T| \neq 1$, then $L_{e}(T)$ is empty. By Lemma 3.7, if $|T|=0$, then $L_{e}(T)=\emptyset$. Suppose $\mathbf{k}$ is a non-special height-one point of $X$. Without loss of generality, we view $\mathbf{k}$ as an upper to $(0)$ in $R[x]=R[s / t]$. If $\mathbf{k}$ is a maximal ideal in $R[x]$, then $\mathbf{k}$ is contained a unique height-two point of $X$, namely $\left(P_{1}, 1 / x\right)$. If $\mathbf{k}$ is not a maximal ideal in $R[x]$, then it follows from Proposition 2.8 that $\mathbf{k}$ is contained in a unique upper to $P_{1}$ and furthermore $\mathbf{k}$ is an integral upper to (0). Thus $\mathbf{k}$ cannot be contained in the point $\left(P_{1}, 1 / x\right)$. If $T=\{\mathbf{p}\}$, then $\left|L_{e}(T)\right|=r$ follows from Theorem 2.4. Thus if $\left(R, P_{1}\right)$ is Henselian, then $X$ satisfies (P5) of $H \mathbb{P}\left(r, k_{1}\right)$.

\section{ACKNOWLEDGEMENT}

It is a pleasure to thank S. McAdam, R. Heitmann, D. Rush, R. Guralnick and the referee for suggestions concerning this paper. 


\section{REFERENCES}

[HLW] W. Heinzer, D. Lantz, and S. Wiegand, Projective lines over one-dimensional semilocal domains and spectra of birational extensions, Proceedings of Conference on Algebraic Geometry and Its Applications, Springer, New York, 1994, pp. 309-325. CMP 94:11

[HW] W. Heinzer and S. Wiegand, Prime ideals in two-dimensional polynomial rings, Proc. Amer. Math. Soc. 107 (1989), 577-586. MR 90b:13010

[HM] R. Heitmann and S. McAdam, Comaximizable primes, Proc. Amer. Math. Soc. 112 (1989), 661-669. MR 91j:13005

[K] I. Kaplansky, Commutative rings, The University of Chicago Press, Chicago, IL, 1974 MR 49:10674

[Ku] E. Kunz, Introduction to commutative algebra and algebraic geometry, Birkhäuser, Boston, MA, 1985. MR 86e:14001

[Ma] H. Matsumura, Commutative ring theory, Cambridge University Press, Cambridge, 1986. MR 88h:13001

[M1] S. McAdam, Intersections of height two primes, J. Algebra 49 (1977), 315-321. MR 58:644

[M2] Uppers in $R[x]$, Comm. Algebra 22 (1994), 1349-1362. MR 94j:13007

[MS] S. McAdam and C. Shah, Substructures of Spec $R[X]$, J. Algebra (to appear).

[N] M. Nagata, Local Rings, Interscience, New York, 1962. MR 27:5790

[S] C. Shah, Prime ideals lying over zero in polynomial rings, J. Algebra 175 (1995), 188-198.

[W] R. Wiegand, The Prime spectrum of a two-dimensional affine domain, J. Pure Appl. Algebra 40 (1986), 209-214. MR 87d:14002

Department of Mathematics, University of Southern California, Los Angeles, CalIFORNIA 90089-1113

Current address: Department of Mathematics, University of California, Riverside, California 92521

E-mail address: cshah@ucrmath.ucr.edu 